\title{
Estimation of the stress-strain state of ultra-low pressure tires
}

\author{
Vladimir Boykov ${ }^{1, *}$, Cheslav Zhdanovich ${ }^{1}$, and Andrey Dzioma ${ }^{1}$ and Joanna Wilczarska ${ }^{2}$ \\ ${ }^{1}$ Belarusian National Technical University, Department "Tractors", 220013 Minsk, Nezavisimosti, 65, \\ Belarus \\ ${ }^{2}$ Faculty of Mechanical Engineering UTP in Bydgoszcz, Department of Vehicle Engineering, Al. \\ Prof. S.Kaliskiego 7, 85-796 Bydgoszcz, Poland
}

\begin{abstract}
To study the stress-strain state and evaluate the operability and durability of the ultra-low pressure tire F118A ns2, its three-dimensional model was developed on the basis of which a finite element model was developed, load regimes were established, and calculations were performed. We consider such modes as tire landing on the disk, pumping the tire to the nominal pressure, loading the tire with the weight of the chassis taking into account contact with the support surface to determine the static contact spot, rolling the tire at a constant speed, and analyzing the non-stationary rolling.
\end{abstract}

\section{Introduction}

Agricultural machinery with its own wheeled propellers and used on fields, meadows and pastures should meet both the requirements of agrotechnics (the pressure of propulsors on the soil and the normal mechanical stress in the soil should not exceed the permissible values), and the requirements for ensuring the specified tire life (tire deformation under action load on the wheel should not exceed the normative values) [1]. The pressure on the soil in the early spring period with its moisture content of $0.9-1.0 \mathrm{HB}$ should not exceed $40 \ldots 50 \mathrm{kPa}[2-4]$. For this purpose, ultra-low pressure tires with an operating air pressure of $10-60 \mathrm{kPa}$ are used on light transport-technological aggregates of agricultural purpose working on the field in the early spring period. When such tires with an air pressure of 10 $15 \mathrm{kPa}$ are fixed, creases are formed (Fig. 1) [5], which leads to their intensive wear. With insufficient internal pressure, the tire loses its carrying capacity, crushing the tire.

The performance and durability of ultra-low pressure tires depends significantly on their load-bearing capacity. In the complex design of pneumatic tires, there are stress concentration zones that can cause premature failure during operation. Fatigue failure leads to a decrease in the life of tires to $20-25 \%$ of the estimated value of the resource for wear. Therefore, analysis of stress-strain state and prediction of longevity in zones of stress concentration of pneumatic tires at the design stage is an actual and important problem [6]. To analyze the stress-strain state of tires, the finite element method [6 - 9] is widely used, which makes it possible to model a complex design of the tire.

\footnotetext{
*Corresponding author: boykov_vip@mail.ru
} 


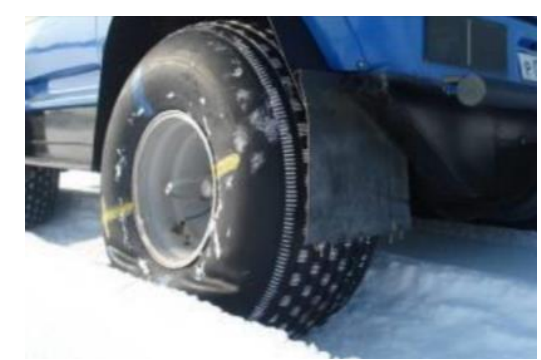

Fig. 1. Formation of folds on tires at a pressure and load of $350 \mathrm{~kg}$ [5].

\section{Main part}

Based on the finite element method using modern software tools, a methodology has been developed and the load capacity of ultra-low pressure tires has been calculated. The model of the model F118A ns2 (22,0 / 70-20) was chosen as the modeling object. The tire has a two-layer textile cord. Construction of the general geometry of the three-dimensional model of the bus was carried out in the software package Creo (Pro / ENGINEER) on the basis of drawings of the section of the tire and the profile of the tread. The main structural elements of the bus F118A ns2 are the frame, bead, belt, protector, sidewall. The construction of each constructive element was carried out in the "Detail" environment, the positioning of each element was carried out in the "Build" environment.To create a common geometry of the tire and tread, a sketch of a repeating tread element was constructed, then using the "Pull" command, each element was constructed. To create a certain number of elements of the tread pattern, the command "Array" was used. Using the "Toroidal bend" command, the flat geometry of the tread was bent according to the shape of the tire. Using the "Rotate" command, the form of the finished tire after vulcanization is given on the basis of the crosssection of the tire of the model.

For three-dimensional modeling of individual layers of the tire, we use the "Rotate" command and a cross-sectional drawing of the tire, which indicates the location of each layer in the body of the tire, creating each layer separately by rotating its section 360 degrees relative to the axis of the tire. The construction of the "Side Ring" part was carried out using the "Stretching" command, knowing the number of turns and the diameter of the wire. Ribbons that cover the joints of the bead ring are built using the "Rotate" command, while the angle to which the section sketch is rotated is determined from the condition that their length is $100 \mathrm{~mm}$. When constructing the carcass plies and the carcass ply, we also took into account the arrangement of the layers in the zone of the bead ring. Figure 2 shows the $3 \mathrm{D}$ model of the tire. 


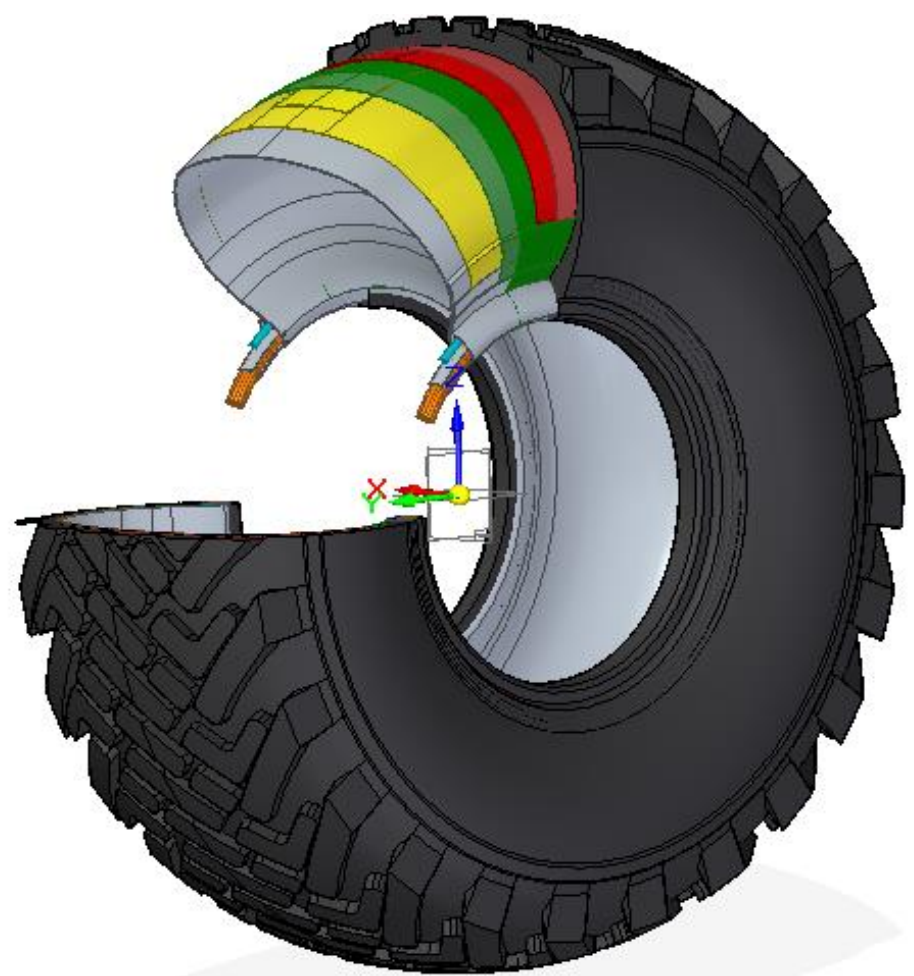

Fig. 2. Three-dimensional model of the bus, taking into account the layers.

In the study of stress-strain state (SSS) tires considered the following main tasks:

1) putting the tire on the disk;

2) pumping the tire to nominal pressure;

3) loading of the tire with the weight of the machine, taking into account contact with the support surface to determine the static contact spot;

4) rolling the tire at a constant speed, while you can get results for effort and torque on the spindle;

5) analysis of non-stationary rolling.

The methodology for solving the tasks is similar for the known universal software complexes used for the study of SSS models, such as ABAQUS, MSC.Software, ANSYS, etc.

Each subsequent step uses the results obtained at the end of the previous step. Whenever possible, it is recommended to use design symmetry to reduce the size of the design model and increase the computing efficiency. 


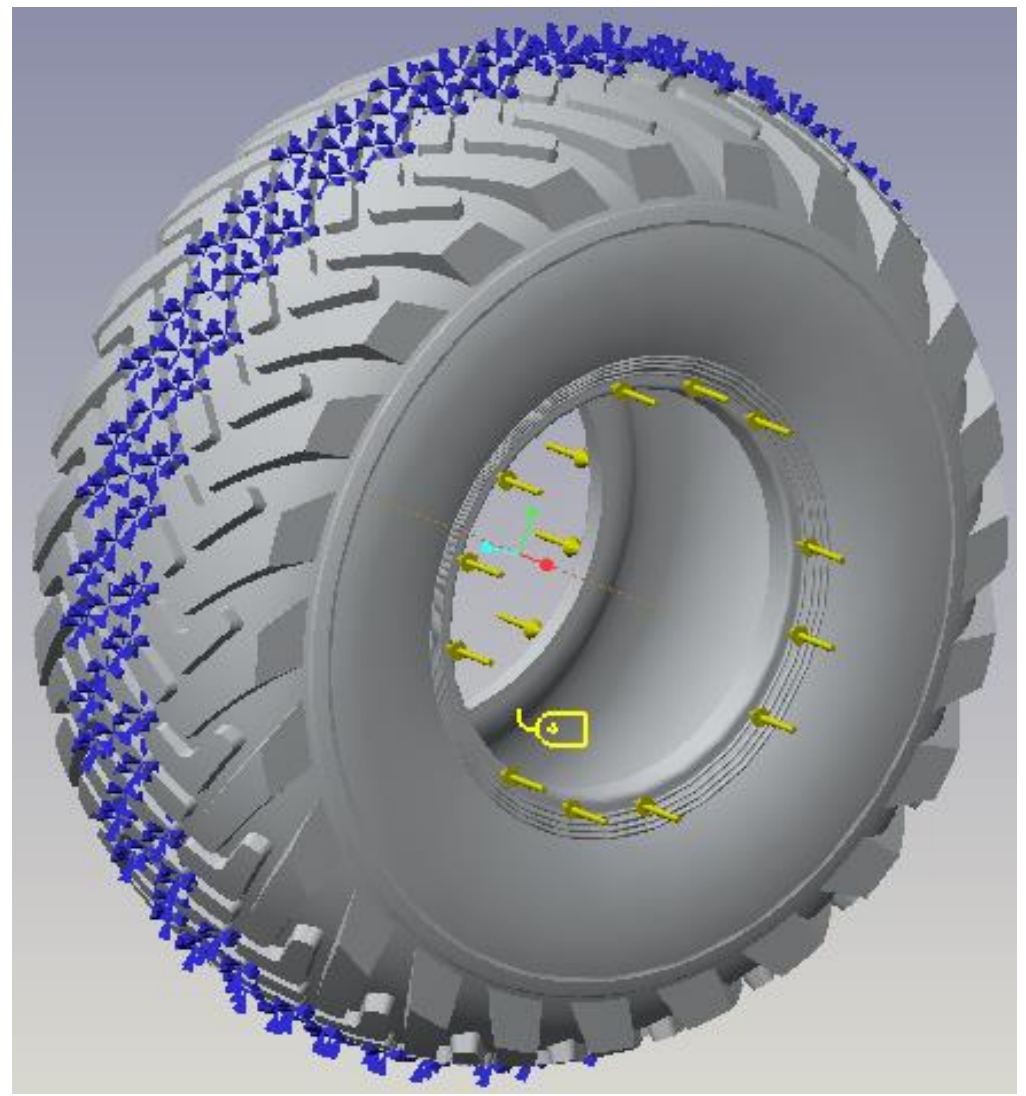

Fig. 3. The scheme of application of loads for the study of SSS when the tire is placed on the disk.

\section{Task of tire landing on the disk.}

In the study of the first problem, a uniform load is applied to the tire surface in the area of the bead ring placement, while the axis of the tire itself remains stationary.Load is applied until the deformation of the tire exceeds the values determined by the parameters of the rim. Further, the value of the applied force becomes zero and the tire tends to return to its original position. Restriction of its movement will be the surface of the rim of the disc.

This task to reduce the calculation time can be solved in a flat section of the bus, and the results can be propagated to the entire circumference of the tire by means of transfer values.

The scheme of application of loads for the study of SSS when landing the tire on the disk is shown in Figure 3.

\section{Pumping the tire to nominal pressure.}

The scheme of application of loads and restrictions for the investigation of SSS when the tire is pumped up to the nominal pressure after landing it on the disk is shown in Figure 4. In this case, the movement restrictions are superimposed on the tire surface in contact with the rim of the disk, and the internal surfaces are loaded with uniformly distributed pressure along the internal surfaces tires. The pressure value during the analysis varies from initial to nominal. 


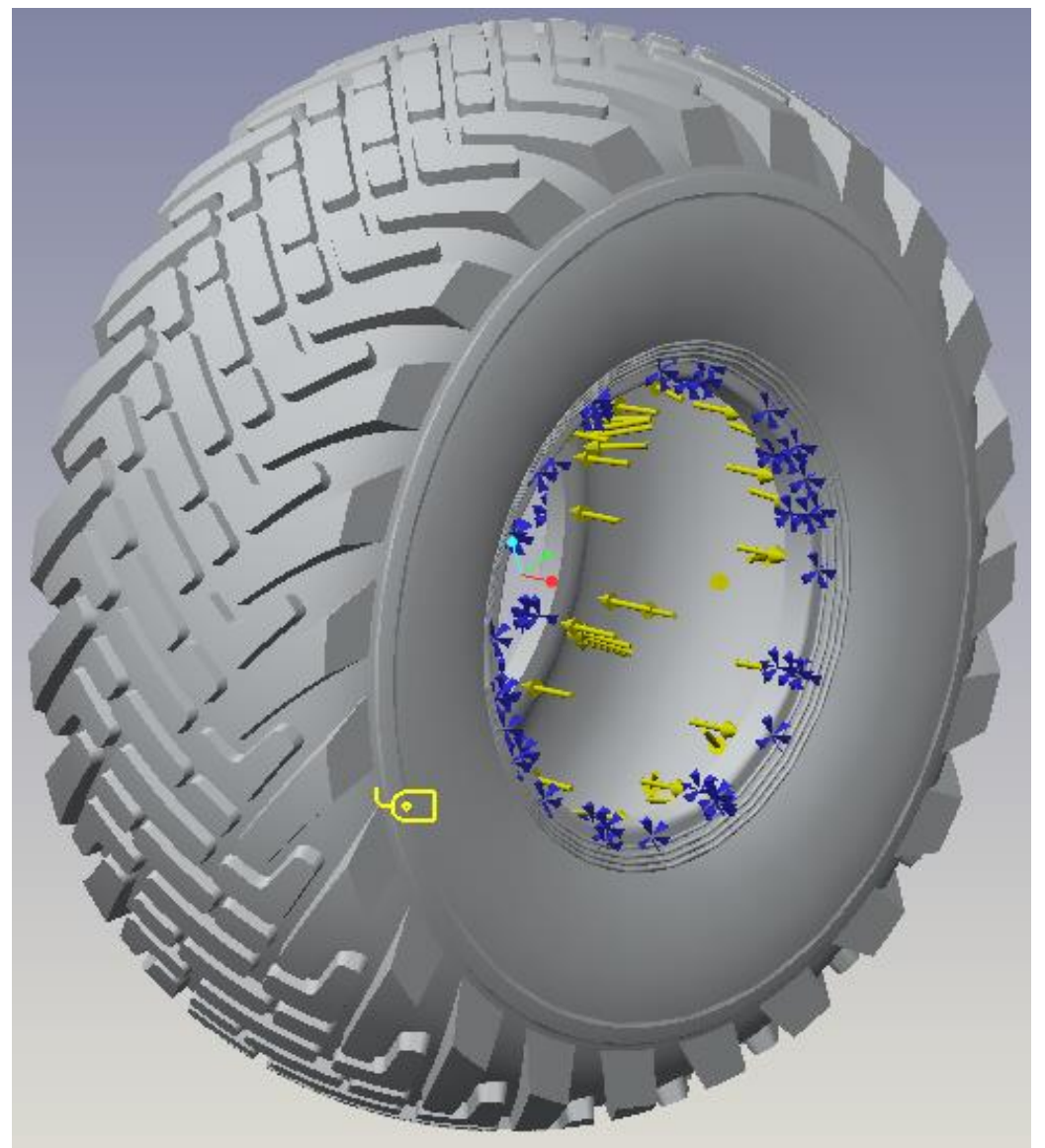

Fig. 4. The scheme of applying loads and imposing constraints in the study of the problem of pumping the tire to the nominal pressure.

Loading of the tire with the weight of the chassis, taking into account contact with the supporting surface for determining the static contact patch.

To solve the task, unlike the previous one, an additional plane is used, loaded so that the magnitude of the applied force at each point of the plane corresponds to the load from the weight of the chassis. The scheme of application of loads and restrictions for the investigation of SSS when the tire is pumped up to the nominal pressure after landing it on the disk is shown in Figure 5.

To study the tire's SSS under the influence of lateral forces, the results of the study of the static contact patch are used and the restrictions on the movement of the tire in the transverse direction are additionally removed and the load applied to the center of the wheel is equal to the lateral load.

The task of rolling a tire is simulated by using a coordinate system associated with the tire axis. The coordinate system moves together with the tire axis, but does not rotate, while the tire rotates relative to the given coordinate system. 


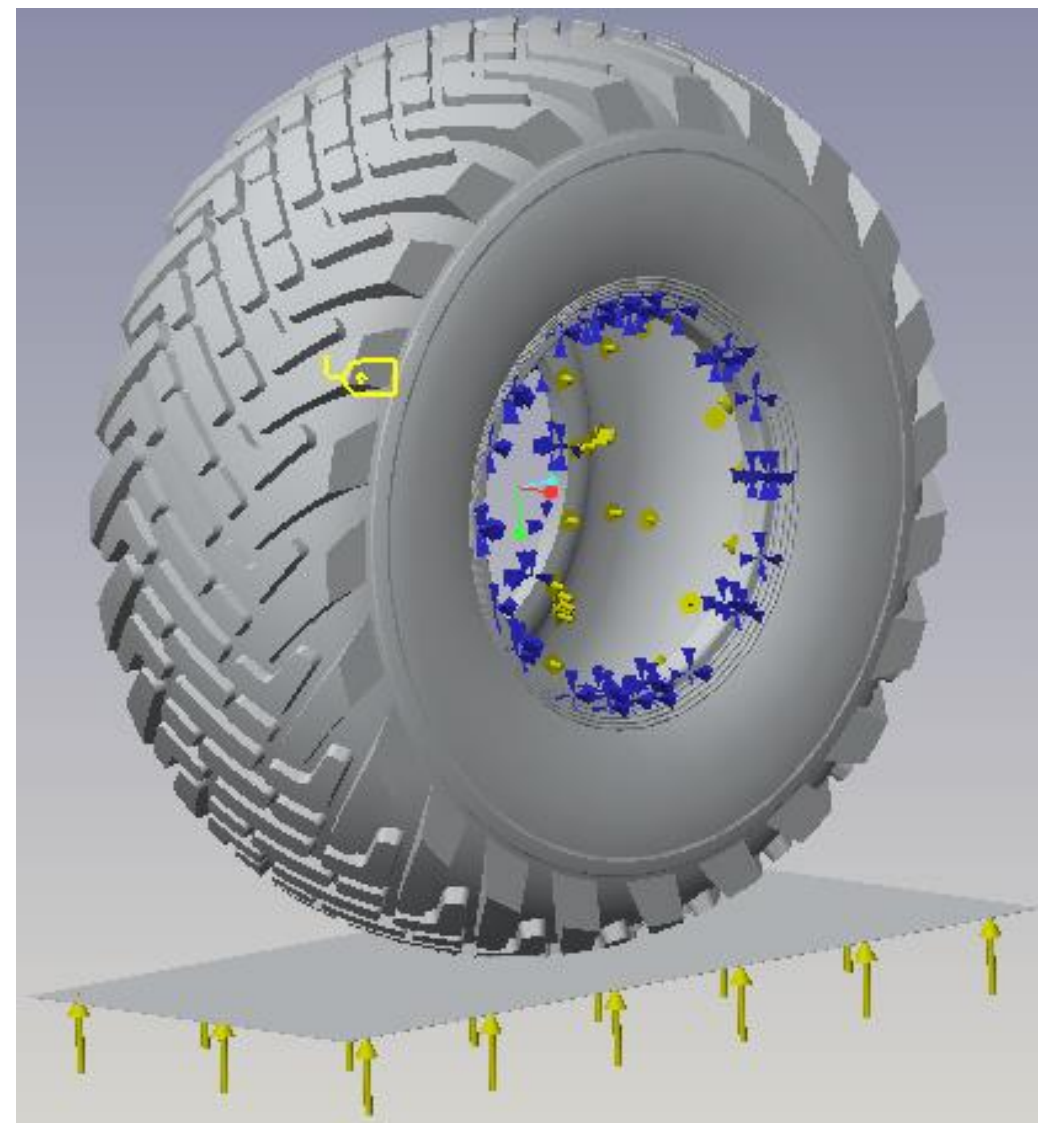

Fig. 5. The scheme of applying loads and imposing constraints in the study of the problem of determining the static contact spot.

For the calculation of the stress-strain state of a tire based on a three-dimensional model, it is necessary to develop an adequate finite element model (FEM). When designing a finite element model (Fig. 6), the type of the problem being solved is taken into account to study the stress-strain state of the tire. For example, to solve the problem of determining the static contact patch, a more detailed grid was formed in the lower part of the tire, and for the stationary rotation problem a detailed grid is only needed near the zone of the contact spot.

Before the construction of the finite element model, materials for each element were assigned. The main material in the tire are rubber compounds. Rubber refers to hyperelastic materials with the inverse convexity of the tension-compression curve. To describe the properties of the real material, the density of the material was set and the data obtained on the basis of the experiment were introduced. They can be specified as a table in the preprocessor by default or through a separate file. In the table, points (nominal stresses and deformations corresponding to them) are introduced, along which the stretching diagram for rubber is constructed (Fig. 7). 


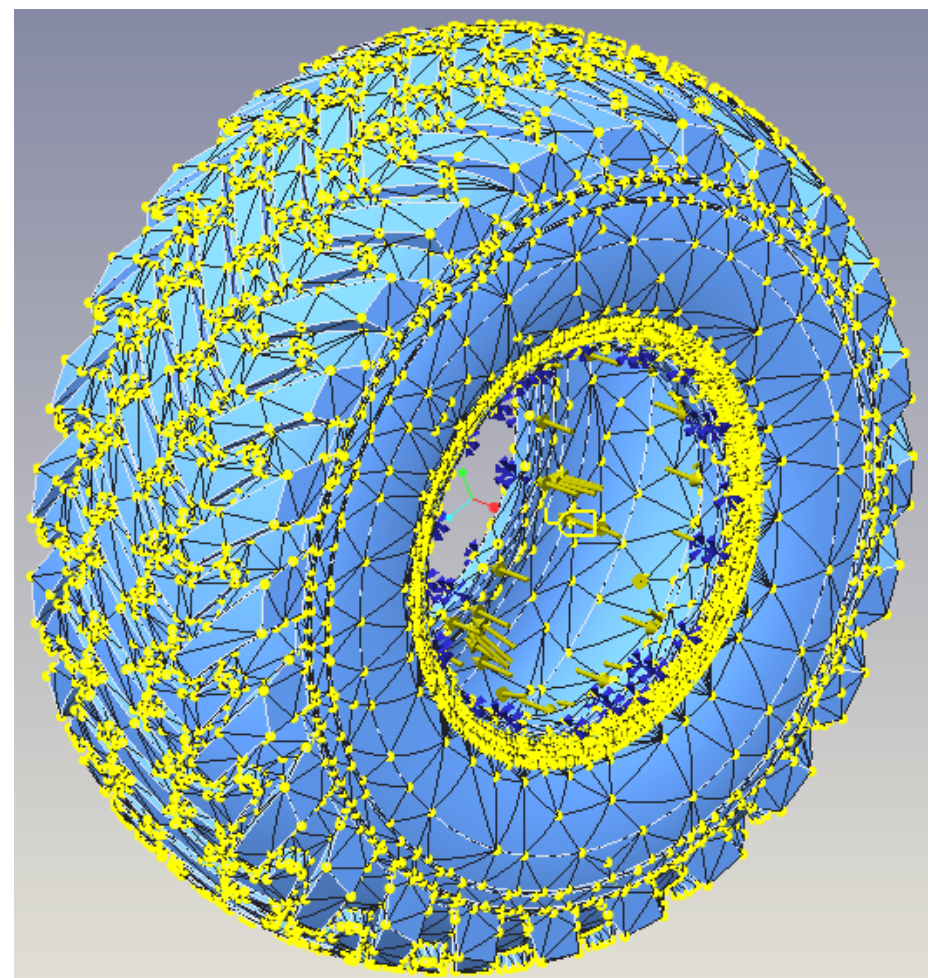

Fig. 6. Automatically created FEM based on the tetrahedral elements.

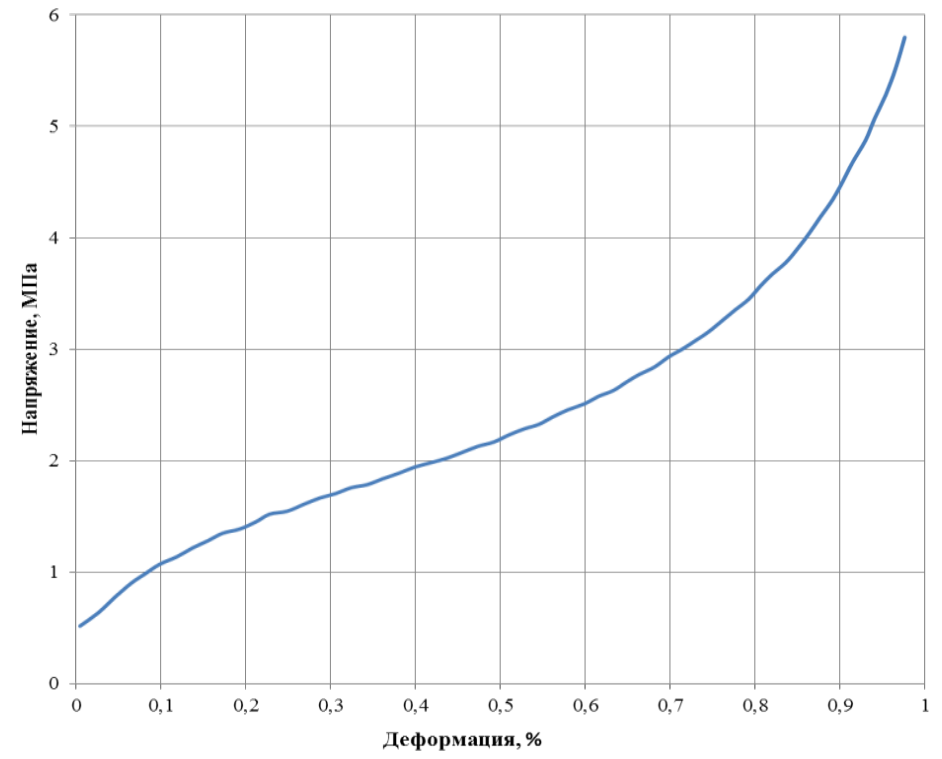

Fig. 7. General view of the rubber extension diagram.

Thus, to study the stress-strain state and evaluate the operability and durability of the ultra-low pressure tire F118A ns2, its three-dimensional model was developed on the basis of which a finite-element model was developed, loading regimes and calculations were performed. The basic operational parameters of the tire are established: the permissible load 
is $6500 \mathrm{~N}$; the maximum load is $8000 \mathrm{~N}$; the tire pressure corresponding to the maximum permissible load is $15 \ldots 20 \mathrm{kPa}$; the maximum speed of movement on unpaved roads with air pressure in the tire is $25 \mathrm{kPa}-50 \mathrm{~km} / \mathrm{h}$.

\section{References}

1. V.A.Rusanov, V.N. Belkovsky. Rubber and Rubber, 9, 6 (1985)

2. V.A.Rusanov, The problem of soil re-consolidation by propulsors and effective ways of its solution (Moscow: VIM, 1998)

3. A.G. Bondarev, V.A. Rusanov, P.M. Sapozhnikov, Overconsolidation of arable soils. Causes, consequences, ways of decreasing, 166 (Moscow: Nauka, 1987)

4. V.V. Medvedev, V.G. Tsybulko, Overconsolidation of arable soils. Causes, consequences, ways of decreasing, 173 (Moscow: Nauka, 1987)

5. L.V. Barakhtanov, Creation of an experimental sample of special vehicles of the northern version on ultra-low pressure tires for operation on low-bearing bearing surfaces (Nizhny Novgorod: NSTU, 2012)

6. 8. S.L. Sokolov, Computational and experimental methods for studying the stressstrain state and cyclic durability of pneumatic tires (Moscow, 2011)

7. 9. LB Barakhtanov, VI Kotlyarenko, SE Manianin, IA Sokolov, Proceedings of the Nizhny Novgorod State Technical University. R.E. Alekseeva, 2 (95), 172 (2012)

8. 10. VO Kondrashov, M.Yu. Sokolova, Izvestiya of the Tula State University Natural Sciences, 4, 144 (2015)

9. 11. Ch.I. Zhdanovich, A.A. Dzioma, Science - education, production, economics. Materials of the 15th International Scientific and Technical Conference, 2, 29 (Minsk, BNTU, 2017) 